\title{
Terahertz response of organic amorphous systems: experimental concerns and perspectives
}

\section{J. Sibik \& J. Axel Zeitler}

To cite this article: J. Sibik \& J. Axel Zeitler (2016) Terahertz response of organic amorphous systems: experimental concerns and perspectives, Philosophical Magazine, 96:7-9, 842-853, DOI: 10.1080/14786435.2015.1111528

To link to this article: https://doi.org/10.1080/14786435.2015.1111528

\section{(?) 2016 The Author(s). Published by Taylor \& Francis}

\section{曲 Published online: 26 Nov 2015.}

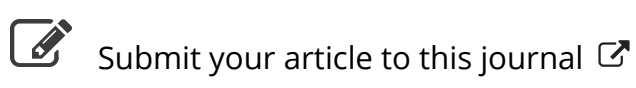

\section{Џll Article views: 817}

View Crossmark data ־

4 Citing articles: 9 View citing articles 


\title{
Terahertz response of organic amorphous systems: experimental concerns and perspectives
}

\author{
J. Sibik* and J. Axel Zeitler \\ Department of Chemical Engineering and Biotechnology, University of Cambridge, Cambridge, UK
}

(Received 6 July 2015; accepted 16 October 2015)

\begin{abstract}
We summarize practical considerations for performing highly accurate terahertz spectroscopy measurements of amorphous systems. We also outline possible perspectives for a further development of terahertz spectroscopy and its applications. This includes a deeper understanding of terahertz dynamics of amorphous systems and the development of a theory for a comparison of terahertz spectra to the results commonly obtained from well-established scattering experiments.
\end{abstract}

Keywords: amorphous; supercooled liqiuids; spectroscopy; terahertz

\section{Introduction}

The formation and properties of amorphous solids remain one of the big unresolved questions in condensed matter physics [1]. A number of experimental techniques have been used to investigate such materials and their complex properties. Commonly used, wellestablished, experimental methods include thermal analysis, dielectric spectroscopy, as well as a broad range of scattering methods such as X-ray, neutron, Brillouin, light and Raman scattering amongst others [2].

Terahertz time-domain spectroscopy (THz-TDS) is one of the more recent analytical techniques to study amorphous systems. The terahertz spectral region refers to the part of the electromagnetic spectrum between microwave and infra-red frequencies. THz-TDS offers direct experimental access to the complex dielectric permittivity at frequencies between $100 \mathrm{GHz}$ and $3 \mathrm{THz}^{1}$ without the need to perform Kramers-Kronig analysis [3], which vastly simplifies the data analysis and makes the technique robust and easy to use. Effectively, THz-TDS can be considered an extension of dielectric spectroscopy to higher frequencies. Compared to dielectric spectroscopy, THz-TDS is however a non-contact method and hence is not limited by practical constrains on performing a high-frequency measurements at low temperatures. Given its frequency range, terahertz spectroscopy directly probes intermolecular motions occurring on pico-second time scales. It is very sensitive to the presence of inter-molecular hydrogen bonds due to the match of the energy between the terahertz photons and the hydrogen bonding energy. Hence, terahertz spectroscopy is an excellent technique to analyse organic compounds in general [4].

We have recently provided THz-TDS evidence on the vitrification of Johari-Goldstein (JG) beta-relaxation in glasses well below the $T_{g}$ [5], and that its onset facilitates the

*Corresponding author. Email: js927@cam.ac.uk 
crystallization of amorphous small organic molecules [6]. A further examination of these results and other high-frequency data on amorphous systems clearly revealed that the vitrification of JG-beta relaxation is a universal process observed across a wide size and chemistry of glass-forming systems, and that the sensitivity of the high-frequency techniques to this effect is linked to the associated changes in the caged dynamics $[2,7,8]$.

Given the lack of pronounced spectral features in terahertz spectra of amorphous systems and the relatively small spectral range that can be measured using THz-TDS compared to dielectric or infra-red spectroscopy, it is very important to perform accurate measurements. To make THz-TDS more widely useful it is critical to better understand the terahertz response of amorphous systems, in particular in the context of the data that can be measured by other, more common techniques. Here we highlight some of the practical aspects that require careful attention when performing terahertz measurements. We also summarize the latest developments in the field of terahertz dynamics of amorphous systems and outline some perspectives for further research in this area.

\section{Experimental concerns}

Given that terahertz spectra of amorphous samples appear featureless, it is extremely important to obtain highly accurate measurements of the absolute complex dielectric function at terahertz frequencies. Great care must be taken to avoid artefacts in the data, e.g. background absorption, which would make it impossible to compare the results with other techniques such as dielectric spectroscopy. Some of the common sources of errors affecting the terahertz results include: (i) air bubbles or macroscopic cracks $(>1 \mu \mathrm{m})$ in the sample; (ii) water uptake into hygroscopic samples; (iii) uneven sample thickness; (iv) discrepancy in windows thickness used for sample and reference (see Figure 1); (v) air gap between reference windows when using two windows as a reference; and (vi) considering real Fresnel coefficients instead of complex ones at optical interfaces with lossy media.

The points (i), (ii) and (iii) can be handled by careful sample preparation. (iv) and (vi) can be accounted for during the data analysis. (v) can either be avoided by ensuring very clean window surfaces or taken into account during the data analysis. We outline further details on performing accurate terahertz experiments of amorphous systems below.

(a)

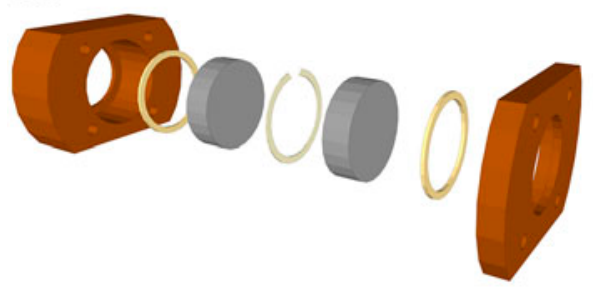

(b)
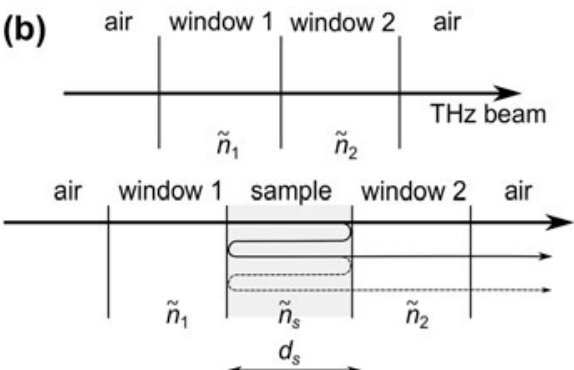

Figure 1. (colour online) (a) Copper sample holder. The windows are pressed together by a copper cradle, with PTFE rings between the quartz and copper to allow differential thermal expansion. The sample is held in the mylar spacer between the windows, with an optional slit cut in it to avoid bubble formation in the centre of the sample. This cradle is then bolted to the coldfinger of the cryostat. After [9]. (b) Corresponding schematic of the reference (top) and sample (bottom) layer structure. Adapted from [10]. 


\subsection{Sample format}

The two most commonly used measurement modalities for solid-state sample THz-TDS in transmission are to prepare a thin film (hundreds of micrometre thick) or a pellet compressed from a powder sample, either from the pure amorphous sample or after mixing with a suitable terahertz transparent diluent such as polyethylene (PE) or polytetrafluoroethylene (PTFE). Preparing a slab of amorphous solid by polishing can be a mechanical challenge due to the brittle nature of the material in organic systems as well as their poor stability towards crystallization. Instead, a sample melt can be loaded into a sample cell consisting of two windows separated by a thin spacer and quench-cooled from the melt as shown in Figure 1. Typically, such a quench-cooled film is the preferred format to achieve highly accurate measurements. It ensures good heat transfer between the sample and the cryostat used for variable temperature measurements given its low (zero) porosity and its relatively thin sample thickness of few tens or hundreds of micrometres. Quench-cooling also generally ensures that the sample is fully amorphous and no nanocrystals remain. On the other hand, quench-cooling is limited when it comes to prepare amorphous mixtures or temperature sensitive samples. From a practical point of view, a further difficulty is to load the melt between two windows such that it is sufficiently sealed to avoid sample leaking before it is solidified during quench-cooling.

A pellet of compressed powder is a sample format that is much easier to prepare and suitable for a wider range of materials. The amorphous powder can be prepared in virtually any way, such as quench-cooling, ball-milling or spray-drying. This is perhaps the most significant advantage compared to the quench-cooled film. However, there are a few limitations as well for this format: the amorphous powder must be stable enough to be pressed into the pellet at room temperature. In addition, the pellet usually disintegrates quickly above $T_{g}$ and hence reliable data can typically only be measured at temperatures below $T_{g}$. A tablet may also adsorb some humidity which affects the results, as discussed below. Given the sample pellet will remain porous during compaction it yields less reproducible results unless it can be prepared in a highly controlled press such as a compaction simulator. When prepared using a manually operated hydraulic press there may be some variations in the sample porosity, hence affecting the value of refractive index [11]. It is important to keep in mind that the compaction process induces pressure stress in the sample and takes several minutes, which limits its suitability for the studies where these factors affect the sample characteristics.

\subsection{Sample thickness}

When preparing the sample, it is important to consider and know its thickness. The sample thickness error propagates the extracted absorption and refractive index and usually represents one of the largest systematic errors of the measurement. Generally, the thicker the sample the smaller the relative error. However, the dynamic range of a typical THzTDS instrument decreases with increasing sample thickness and hence there is a trade-off between frequency bandwidth and the sample thickness [12].

\subsection{Windows}

Particular consideration needs to be given to choosing a suitable window material to sandwich the sample in between that allows quench-cooling, is transparent to terahertz 

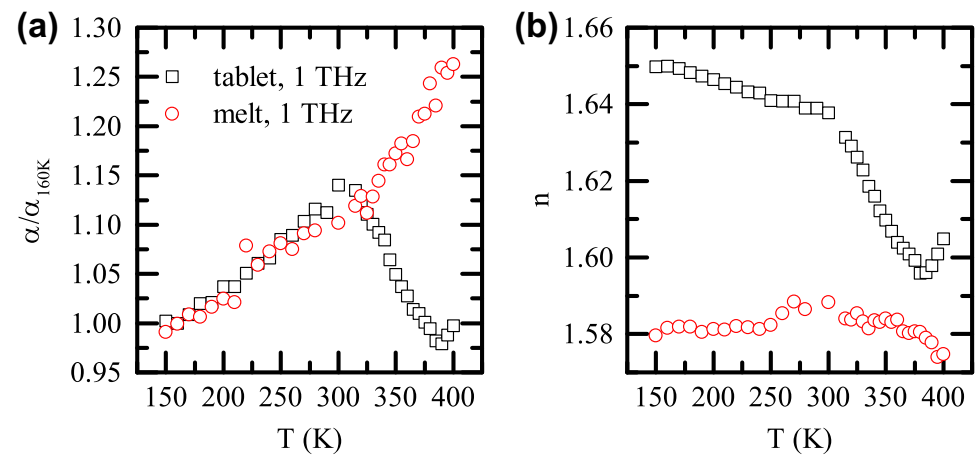

Figure 2. (colour online) (a) Absorption coefficient and (b) refractive index of polyvinylpyrrolidone/vinyl acetate (Kollidon VA 64) at $1 \mathrm{THz}$ prepared as tablet (black squares) and thin film from melt (red circles).

radiation, is rigid over a wide temperature range and provides good heat transfer. Crystalline silica (z-cut quartz) windows are highly transparent to terahertz radiation but prone to shatter upon thermal shock. Amorphous silica windows can sustain fast quench-cooling but absorb terahertz radiation more strongly compared to quartz, and hence limit the dynamic range and in turn decrease the accessible bandwidth of the terahertz spectrometer. Silicon windows are non-absorbing and can sustain thermal shock, but are not transparent at visible frequencies and thus do not allow to check presence of air bubbles in the sample cell which strongly distort the terahertz beam. Also, the refractive index of silicon is very high at terahertz frequencies which would result in further signal attenuation due to high reflective losses at the window interfaces. Polymer windows from high density PE our PTFE are generally not recommended due to their poor transparency at visible frequencies, their poor thermal conduction and high mechanical flexibility, which may result in optical aberrations. In addition to choosing the best possible material for the windows, it is important to take the windows explicitly into account during data processing when extracting the optical properties of the sample material.

\subsection{Humidity and water adsorption}

Great care needs to be given to the humidity of the sample, in particular in the case of relatively porous pellet samples with large surface area, as water strongly absorbs terahertz radiation and amorphous organic materials are typically highly hygroscopic. It is crucial to perform the terahertz measurement under vacuum or nitrogen atmosphere to maximize the terahertz signal and avoid absorption due to the rotational transitions in water vapour. Many polymers and the sample materials themselves are hygroscopic and may adsorb water up to the equivalent of few per cent of mass. We would like to emphasize that in the following we refer to the bulk water that is adsorbed by the surface of the pores in the sample and not any water dispersed with the sample at a molecular level. Any adsorbed water will influence the obtained terahertz results. This is demonstrated in the example of amorphous polymer in Figure 2, where we show the absorption coefficient and the refractive index at a frequency of $1 \mathrm{THz}$ as a function of temperature for the case of a tablet with adsorbed water and a quench-cooled melt sample with no adsorbed water. 
The absorption coefficient generally shows a comparable relative change upon heating in both samples up to temperatures of about 300 , where the samples start to exhibit opposite trends. In the case of the quench-cooled melt sample, the absorption increases further with temperature as would be expected theoretically [5], while the refractive index stays roughly constant. In contrast to the pellet sample that contains adsorbed water, both the absorption coefficient and refractive index drop at temperatures above $300 \mathrm{~K}$. This can be explained by water boiling and its subsequent evaporation, since the boiling point of water is $\approx 26^{\circ} \mathrm{C}=$ $299 \mathrm{~K}$ at $30 \mathrm{mbar}$, the pressure at which the experiment was performed. Loss of bulk water inevitably lowers the effective absorption coefficient of the sample. Given that the refractive index of water is approx. 2.2 [13], while that of the polymer is approx. 1.6 (see Figure 2(b)), loss of water will also result in a drop of the measured refractive index. Once the water escapes, the refractive index of the pellet and the quench-cooled melt are of approximately the same value as can be seen in our example at $380 \mathrm{~K}$.

It is possible to avoid the undesired effect of humidity by (i) careful handling of the sample and avoiding exposure to ambient humidity by working under a dry nitrogen atmosphere, (ii) storing the sample in a dessicator once it is prepared or (iii) exposing the sample to vacuum at an elevated temperature (yet below $T_{g}$ ) for the time necessary to dry the sample, if the studied material has a sufficiently high $T_{g}$ and the associated ageing of the sample can be neglected. It should be emphasized that the case presented in Figure 2 is an extreme example of the effect of water loss on terahertz spectra.

\subsection{Data analysis}

In order to compare the terahertz results with dielectric spectroscopy, it is necessary to convert the refractive index and absorption coefficient into the real and imaginary parts of the dielectric function:

$$
\begin{aligned}
\tilde{n}(v) & =n(v)+i \kappa(v) \\
\kappa(v) & =\alpha(v) c /(4 \pi v) \\
\tilde{\epsilon}(v) & =\epsilon^{\prime}(v)+i \epsilon^{\prime \prime}(v)=\tilde{n}(v)^{2} \\
\epsilon^{\prime}(v) & =n(v)^{2}-\kappa(v)^{2} \\
\epsilon^{\prime \prime}(v) & =2 n(v) \kappa(v),
\end{aligned}
$$

where $\tilde{n}$ is the complex refractive index as measured by THz-TDS, $n$ is the real refractive index, $\kappa$ is the extinction coefficient, $\alpha$ is the absorption coefficient, $v$ is the linear frequency, $c$ is the speed of light in vacuum, $\tilde{\epsilon}$ is the complex dielectric function, $\epsilon^{\prime}$ is the real dielectric permittivity and $\epsilon^{\prime \prime}$ is the dielectric loss. A reliable method for extraction of optical constants from terahertz measurements has been outlined by Duvillaret et al. [10]. Their method offers a very robust implicit way of extracting optical constants, posing virtually no limitation on the sample structure. It can be adapted easily to single slab samples as well as samples sandwiched between windows. In this context, it is important to consider the complex refractive indices of both windows (or surrounding media in general) and the sample in the data analysis. This is absolutely critical in order to obtain sufficient accuracy in the terahertz results to compare with dielectric spectroscopy. Explicit expression of the absorption coefficient and the refractive index using the Beer-Lambert law, such as Equations (1) and (2) in [12], is not suitable as it may lead to an offset in 


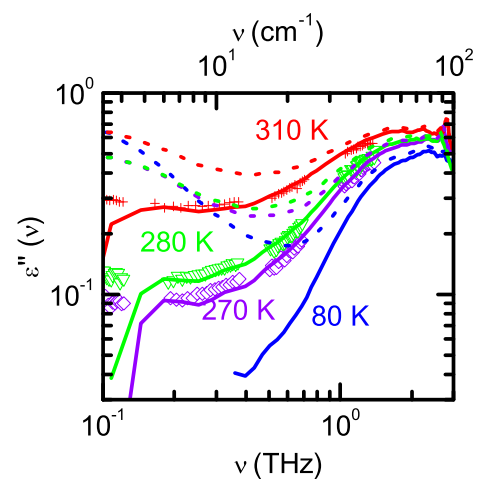

Figure 3. (colour online) Comparison between dielectric and terahertz spectroscopy results of amorphous sorbitol. Data represent dielectric spectroscopy measurement [14]. Solid lines are terahertz data extracted by the method of Duvillaret et al. [10] and considering the complex refractive index of windows and sample. Dashed lines are based on the explicit calculation of the terahertz optical properties using Beer-Lambert law, resulting in a positive absorption coefficient offset of few $\mathrm{cm}^{-1}$ and consequent wing in the dielectric losses at low frequencies. Adapted from [5].

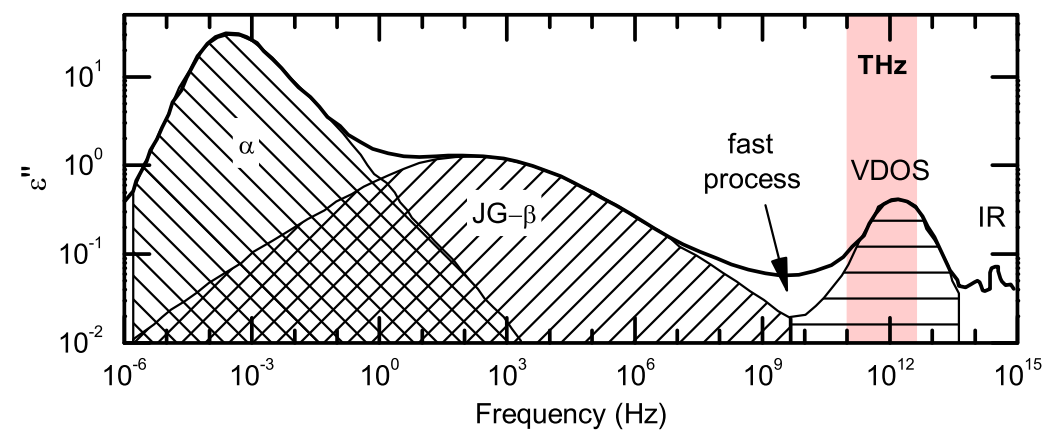

Figure 4. (colour online) Schematics of different relaxations and vibrations in dielectric spectra of amorphous materials. Adapted from http://www.physik.uni-augsburg.de/lehrstuehle/exp5/themen/ dismatter/glassfl/.

the absorption coefficient by neglecting the imaginary part of the Fresnel coefficients at the optical interfaces. Such background absorption will lead to an arbitrary increase in the dielectric losses $\epsilon^{\prime \prime}$ in the limit of $0 \mathrm{THz}$ (see Equations (1b) and (1e)), due to the division of a constant by zero. This is demonstrated in Figure 3.

\section{Perspectives}

THz-TDS is a new technique to gain direct insight into the changes in amorphous systems at the inter-molecular level. In the following part we highlight some of the phenomena that can be observed in the glassy state and outline a number of perspectives for future research.

\subsection{Temperature effect on terahertz dynamics}

Generally, the terahertz dynamics of supercooled liquids contains a contribution from the fast molecular relaxations as well as vibrations or librations of the molecules, the latter 
being described by the vibrational density of states (VDOS) and resulting in a broad spectral feature in the dielectric loss between approximately $0.1-5 \mathrm{THz}$ or so (see Figure 4). We will refer to this broad feature as the VDOS peak, to avoid its misinterpretation as the boson peak. The term boson peak refers only to the part of the VDOS in excess of the Debye level [15], while the VDOS peak covers the complete part of the VDOS that can be excited at terahertz frequencies.

The temperature behaviour of terahertz dynamics shows certain similarities for all amorphous systems. When cooling a liquid, two thermal events can be resolved by the response of the absorption at terahertz frequencies to the change in temperature: at $T_{\alpha} \approx T_{g}$ and $T_{\beta}<T_{g}$ [5]. These events strongly coincide with the vitrification temperature of $\alpha$ and the JG $\beta$-relaxation, respectively [2]. Generally, $T_{\alpha}$ and $T_{\beta}$ can be determined from terahertz data by a simple linear fit of $\alpha_{\mathrm{THz}}(T)$ or $\epsilon_{\mathrm{THz}}^{\prime \prime}(T)$ [5].

Perspective 1 (Non)linearity of terahertz absorption with temperature.

For relatively simple small organic molecules $\alpha_{\mathrm{THz}}(T)$ or $\epsilon_{\mathrm{THz}}^{\prime \prime}(T)$ change with temperature in a linear fashion for $T>T_{g}$ and $T_{g}>T>T_{\beta}$, as previously reported [5]. It is however not immediately obvious why the change in absorption follows the empirically observed linear trends. The $\alpha$-relaxation time $\tau_{\alpha}(T)$ generally follows Vogel-FulcherTammann dependence at $T>T_{g}$, while the JG $\beta$-relaxation time $\tau_{\beta}(T)$ follows Arrhenius dependence at $T_{g}>T>T_{\beta}$. Not only are these two characteristics far from the linear trend in the mathematical sense, they are also quite distinct from each other. It has been recently discussed that it is not likely for the $\alpha$ and JG $\beta$-relaxation to directly contribute to terahertz dynamics close to $T_{g}$ and $T_{\beta}$, respectively. Instead, the vitrification of the dielectric relaxations appears to affect the terahertz response via the cage dynamics, or nearly constant losses (NCL) $[2,16,17]$. This explanation still does not explain the linearity of $\epsilon_{\mathrm{THz}}^{\prime \prime}(T)$ nor does it explain why the NCL amplitude is proportional to the changes in the amplitude or relaxation time of the $\alpha$ and $\mathrm{JG} \beta$-relaxations, as a simple addition is certainly not a suitable explanation given the difference in the thermal behaviour that is observed for $\tau_{\alpha}(T)$ and $\tau_{\beta}(T)$.

Hypothetically, this may mean that the NCL region behaves somewhat independent of the actual relaxation times $\tau_{\alpha}$ and $\tau_{\beta}$. The change in the caged dynamics at $T_{g}$ and $T_{\beta}$ must then reflect on a change in the actual cages. This would inherently mean that despite the strong kinetic changes during the vitrification, there are related structural changes occurring at $T_{g}$ and $T_{\beta}$ in the glass. While such conclusion is highly hypothetical at the present time, it would explain the high sensitivity of terahertz spectroscopy to the presence of $T_{g}$ and $T_{\beta}$, given that terahertz spectroscopy directly probes the inter-molecular vibrations and is extremely sensitive to any changes in the local molecular structure, as has been already demonstrated on the case of liquids [18].

In more complex and flexible disordered molecular systems, such as disaccharides and proteins, the terahertz response does not exhibit the simple linear response with temperature that was described above. As can be seen from Figure 5, the behaviour in $\alpha_{\mathrm{THz}}(T)$ at $T<T_{g}$ is deviating from two linear curves and instead a more gradual change with temperature is observed as the complexity of the molecule increases. A likely reason for this is the interference of non-JG relaxations [20], such as those originating from internal flexibility of the molecule, with the terahertz dynamics. This also needs to be investigated in greater detail in future studies. 


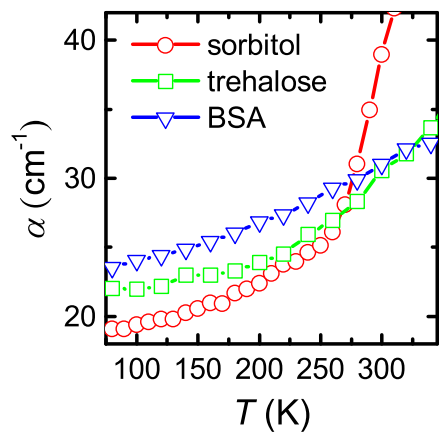

Figure 5. (colour online) Terahertz absorption of amorphous sorbitol, trehalose and BSA between 80 and $340 \mathrm{~K}$ at $0.8 \mathrm{THz}$. Adapted from [19].

Perspective 2 Temperature change of the VDOS peak.

In inorganic glasses, the temperature effect on the VDOS is usually negligible [21], but this is not the case for organic glasses. An example can be seen in Figure 6, where standard THz-TDS results have been extended to higher frequencies by a combination of the TDS data with those obtained using the far-infrared beamline B22 at the Diamond Light Source following Kramers-Kronig analysis for a sample of amorphous sorbitol [22]. At its higher frequency end the VDOS peak appears to have some internal structure with two local maxima observed at $100 \mathrm{~K}$ around 2.5 and $3.6 \mathrm{THz}$. Further peaks are observed at 8 and $10 \mathrm{THz}$, originating likely from intra-molecular vibrations given their high frequency. Closer analysis indicates that at temperatures well below $T_{g}$ the VDOS first shifts to slightly lower frequencies upon heating. It then broadens significantly above $T_{g}$. We therefore conclude that the thermal changes observed in the terahertz spectra are a combination of the changes in the dielectric relaxations and the VDOS peak. The data however also show that at frequencies below $2 \mathrm{THz}$ the dielectric relaxations have a much stronger effect, especially above $T_{g}$. In order to carefully analyse the temperature shift of the VDOS it is necessary to improve the quality of the data e.g. using a THz-TDS instrument with a bandwidth of $>4 \mathrm{THz}$ instead of combining the spectra of two separate measurements, and to subtract the relaxation contributions from the terahertz data by extrapolation of high-frequency dielectric spectroscopy data.

\subsection{Comparison of terahertz spectroscopy to scattering techniques}

It has been reported that the major contribution to the terahertz absorption of amorphous solids originates from the coupling of terahertz radiation to the VDOS [23]. Terahertz spectroscopy is by far not the only technique that can be used to measure the VDOS. Similar measurements can be obtained by methods such as neutron scattering, light scattering, Brillouin scattering, low-frequency Raman scattering and optical heterodyne detected optical Kerr effect techniques [2]. The advantage of THz-TDS is its simplicity and robustness of use, with minimal practical constraints on performing the experiments in a laboratory setting, straightforward and accurate data extraction and the availability of commercial terahertz spectroscopy systems.

In order to fully appreciate the terahertz response of amorphous systems, it is necessary to develop mathematical tools for a direct comparison of the THz-TDS data to that acquired 


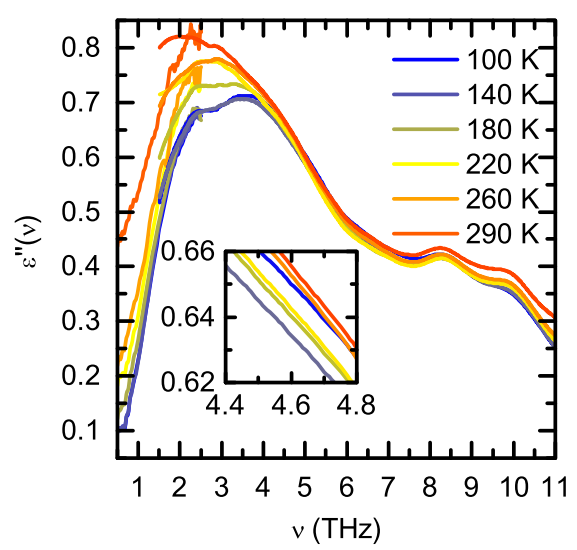

Figure 6. (colour online) Dielectric losses of amorphous sorbitol observed by a combination of terahertz spectroscopy and Diamond Light Source [22]. Inset shows a detail of the spectra between 4.4 and $4.8 \mathrm{THz}$.

using the other techniques mentioned above. It may be easiest to compare the terahertz measurements to low-frequency Raman measurements for which the suitable theoretical description already exists [24]. However, such a model needs to take into account the difference in the nature of the response: while the Raman signal originates from the polarizability of the molecules, the terahertz spectroscopy response originates from the molecular dipole moment. This task remains to be addressed in the future. To stimulate such a development we highlight some of the similarities that are apparent from the experimental data.

\section{Perspective 3 VDOS and the boson peak.}

The boson peak generally refers to the excess in the density of states $g(v)$ above the Debye level $g_{\text {Debye }}(v) \propto v^{2}[15]$. In the simplest first-order perturbation process of photon interaction with atomic vibrations the terahertz absorption coefficient can be expressed as $\alpha(v)=C(v) g(v)$, where $C(v)$ represents the coupling coefficient and $g(v)$ the VDOS [23]. Taraskin et al. showed that at frequencies below the Ioffe-Regel crossover $v \lesssim v_{\mathrm{IR}}$ the general dependence of the coupling coefficient follows $C(v)=A+B v^{2}$, where $A$ and $B$ are material-dependent constants [23].

The far-infrared absorption of glasses often follows $\alpha(v) \propto v^{2}$ [21], which can be explained by the approximation $g(v) \approx g_{\text {Debye }}(v)$ and $C(v) \approx A$. Considering the latter to hold, it is possible to determine the excess to the Debye VDOS by examining $\alpha(v) / v^{2}$. This is shown schematically in Figure 7(a). Using a qualitative assignment the VDOS can be split into (i) a constant level, corresponding to $\propto v^{2}$, i.e. the Debye level; (ii) the low-frequency wing from the relaxation contributions at higher temperatures and (iii) a broad peak centred around $1.5 \mathrm{THz}$ that strongly resembles the boson peak as observed from inelastic X-ray scattering measurements [25]. A similar peak feature can also be observed directly in the refractive index (Figure 7(b)). This behaviour is not too surprising considering that any vibrational resonance has its signature in the refractive index. It is however very interesting to note that the peaks differ in the frequency of their maxima at $80 \mathrm{~K}: v_{\max \left\{\alpha / v^{2}\right\}}=1.55 \mathrm{THz} \cong 6.4 \mathrm{meV}$, while $v_{\max \{n\}}=1.08 \mathrm{THz} \cong 4.7 \mathrm{meV}$. These values seem to correspond with the Ioffe-Regel limit $\Omega_{\mathrm{IR}}=6.7 \mathrm{meV}$ and the boson peak 
(a)

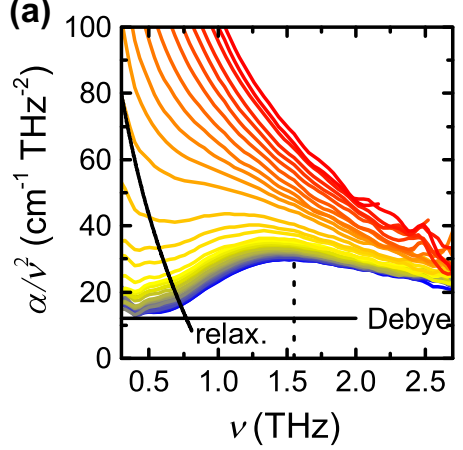

(b)

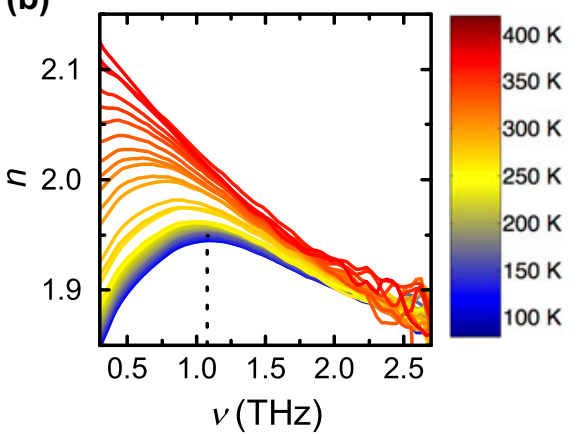

Figure 7. (colour online) Terahertz spectra of amorphous sorbitol between 80 and $420 \mathrm{~K}$ : (a) Rescaled absorption coefficient $\alpha(v) / v^{2}$ and (b) refractive index. The solid lines are plotted to guide the eye and highlight the Debye and relaxational contribution to the rescaled absorption coefficient. The dashed lines highlight the frequency of the peak maximum which is observed at $1.55 \mathrm{THz}$ in (a) and $1.08 \mathrm{THz}$ in (b).

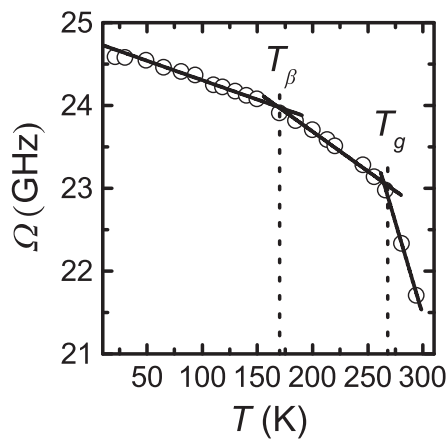

Figure 8. Temperature dependence of the frequency shift of the longitudinal acoustic modes as obtained by Brillouin scattering. The data are taken form [26]. The solid lines represent linear fits. The dashed lines highlight $T_{\beta}=170 \mathrm{~K}$ and $T_{g}=268 \mathrm{~K}$.

centre $\Omega_{\mathrm{BP}}=4.5 \mathrm{meV}$, respectively, as determined in the inelastic X-ray scattering studies [25]. This similarity is striking and certainly requires further examination.

Perspective 4 Relationship of terahertz data to the elastic properties and the mean-squared displacement.

As mentioned before, the terahertz spectroscopy results are expected to provide similar information and complement a range of scattering techniques [2]. The obvious question to ask is whether there is any evidence for $T_{\beta}$ as observed using THz-TDS in these scattering data? In Figure 8 we replot the temperature dependence of the frequency shift in amorphous sorbitol measured by Brillouin light scattering [26]. A careful examination reveals a deviation that occurs at $T_{\beta} \approx 170 \mathrm{~K}$, which is in excellent agreement with our terahertz studies [5] as well as dielectric spectroscopy [2]. The same effect can also be 
detected for the longitudinal sound velocity data (see Figure 4 in [26]), which is not too surprising given the linear relationship between the two properties.

Given that it is possible to observe these effects using THz-TDS, this opens the question whether it is possible to substitute some of the currently used scattering measurements with THz-TDS measurements. This could be particularly useful when considering the practical difficulties in terms of expense and beam time availability to perform neutron scattering measurements, which makes it an unsuitable technique for routine analysis of a large number of samples. As an example, neutron scattering has proven to be useful in determining the dynamical transition of solvated proteins by examining the means square displacement $\left\langle u^{2}\right\rangle$ [27] and it has been since demonstrated that similar information can be extracted using THz-TDS [28,29].

\section{Conclusions}

In conclusion, there are a number of important practical considerations to satisfy in order to perform accurate terahertz measurements of amorphous systems. However, once this hurdle is mastered THz-TDS is a very versatile technique that can be used to investigate a range of very interesting properties of the amorphous state, some of which we have outlined in this paper. We believe that terahertz spectroscopy has a significant potential to complement some of the measurements that are currently performed using far more complicated techniques such as inelastic neutron scattering.

\section{Acknowledgements}

The authors gratefully acknowledge S. Capaccioli, K. Ngai and W. Schirmacher for stimulating discussions, as well as G. Cinque for his assistance with the measurements at the Diamond Lights Source. This work was carried out with the support of the Diamond Light Source. We would also like to acknowledge the UK Engineering and Physical Sciences Research Council [EP/J007803/1] as well as MedImmune for funding. We thank to Bristol-Myers Squibb for the generous donation of Kollidon VA 64. Additional data related to this publication are available at the Cambridge University DSpace repository (https://www.repository.cam.ac.uk/handle/1810/252491).

\section{Disclosure statement}

No potential conflict of interest was reported by the authors.

\section{Funding}

This work was carried out with the support of the Diamond Light Source; the UK Engineering and Physical Sciences Research Council [EP/J007803/1] and MedImmune.

\section{Note}

1. The spectral range that can be accessed by THz-TDS varies depending on the technique used between 0.1 and $3 \mathrm{THz}$ for the most widely used type of setups (photoconductive antennas and optical rectification), $0.5-7 \mathrm{THz}$ for Cherenkov sources or even 0.5 to $>10 \mathrm{THz}$ for air plasma sources. All setups have in common that the dynamic range of the spectrometer drops exponentially with increasing frequency hence limiting the usable bandwidth significantly for strongly absorbing samples. For the purpose of this paper we base our discussion on the range of $0.1-3 \mathrm{THz}$ except where specified explicitly. 


\section{References}

[1] G. Biroli and J.P. Garrahan, J. Chem. Phys. 138 (2013) p.12A301.

[2] S. Capaccioli, K.L. Ngai, M. Thayyil, D. Prevosto, J. Phys. Chem. B 119 (2015) p.8800.

[3] H. Tuononen, E. Gornov, J.A. Zeitler, J. Aaltonen and K.E. Peiponen, Opt. Lett. 35 (2010) p.631.

[4] E.P. Parrott and J.A. Zeitler, App. Spectrosc. 69 (2015) p.1.

[5] J. Sibik, S.R. Elliott and J.A. Zeitler, J. Phys. Chem. Lett. 5 (2014) p.1968.

[6] J. Sibik, K. Loebmann, T. Rades and J.A. Zeitler, Mol. Pharm. 12 (2015) p.3062. doi:10.1021/acs.molpharmaceut.5b00330.

[7] K.L. Ngai, S. Capaccioli, D. Prevosto and L.-M. Wang, J. Phys. Chem. B 119 (2015) p.12502.

[8] K.L. Ngai, S. Capaccioli, D. Prevosto and L.-M. Wang, J. Phys. Chem. B 119 (2015) p.12519.

[9] J. Sibik, M.J. Sargent, M. Franklin and J.A. Zeitler, Mol. Pharm. 11 (2014) p.1326.

[10] L. Duvillaret, F. Garet, and J.L. Coutaz, IEEE J. Sel. Top. Quant. 2 (1996) p.739.

[11] E.P. Parrott, J.A. Zeitler and L.F. Gladden, Opt. Lett. 34 (2009) p.3722.

[12] P.U. Jepsen and B.M. Fischer, Opt. Lett. 30 (2005) p.29.

[13] U. Møller, D.G. Cooke, K. Tanaka and P.U. Jepsen, J. Opt. Soc. Am. B 26 (2009) p.A113.

[14] S. Kastner, M. Köhler, Y. Goncharov, P. Lunkenheimer, A. Loidl and J. Non-Cryst, Solids 357 (2011) p.510.

[15] U. Buchenau, N. Nücker and A.J. Dianoux, Phys. Rev. Lett. 53 (1984) p.2316.

[16] K.L. Ngai, Relaxation and Diffuson in Complex Systems, Springer, New York, 2011.

[17] K.L. Ngai, J. Phys. Condens. Matter 15 (2003) p.S1107.

[18] F.O. Nielsen, Annu. Rep. Prog. Chem. C 90 (1993) p.3.

[19] M.A. Mensink, J. Sibik, H.W. Frijlink, K. van der Voort Maarschalk, W.L.J. Hinrichs, J.A. Zeitler, 2015 ; in preparation.

[20] K.L. Ngai, J. Chem. Phys 120 (2004) p.857.

[21] U. Strom, J.R. Hendrickson, R.J. Wagner and P.C. Taylor, Solid. State. Commun. 15 (1974) p.1871.

[22] J. Sibik, Terahertz spectroscopy of glasses and supercooled liquids, Ph.D. diss., University of Cambridge, Cambridge, 2014.

[23] S.N. Taraskin, S.I. Simdyankin, S.R. Elliott, J.R. Neilson and T. Lo, Phys. Rev. Lett. 97 (2006) p.055504.

[24] B. Schmid and W. Schirmacher, Phys. Rev. Lett. 100 (2008) p.1.

[25] B. Ruta, G. Baldi, F. Scarponi, D. Fioretto, V.M. Giordano and G. Monaco, J. Chem. Phys. 137 (2012) p.214502.

[26] B. Ruta, G. Monaco, F. Scarponi and D. Fioretto, Philos. Mag. 88 (2008) p.3939.

[27] S. Capaccioli, K.L. Ngai, S. Ancherbak and A. Paciaroni, J. Phys. Chem. B 116 (2012) p.1745.

[28] A.G. Markelz, J.R. Knab, J.Y. Chen and Y. He, Chem. Phys. Lett. 442 (2007) p.413.

[29] F. Lipps, S. Levy and A.G. Markelz, Phys. Chem. Chem. Phys. 14 (2012) p.6375. 